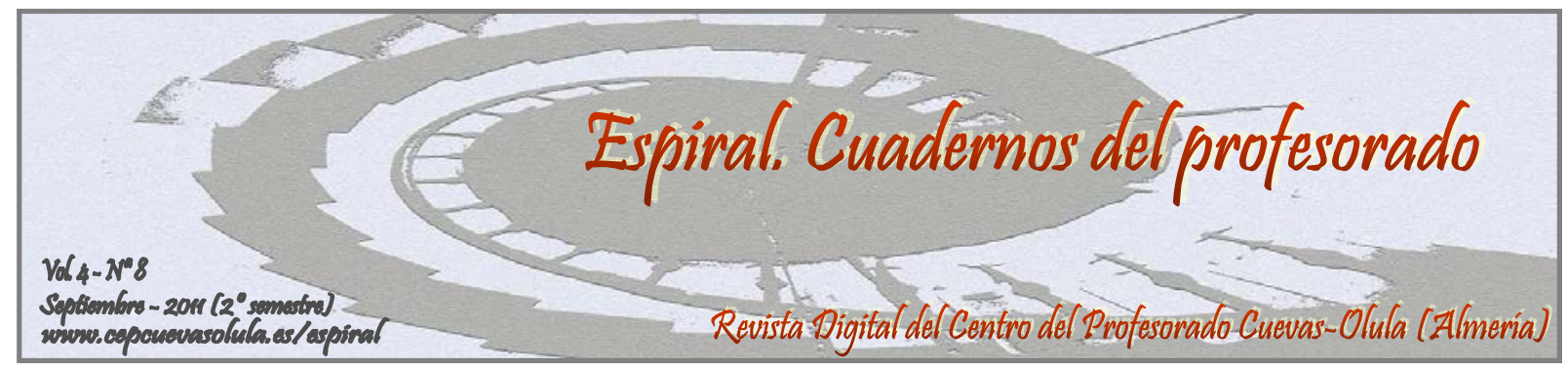

\title{
PRÁCTICA DE PADEL ADAPTADO, UN MODELO DE INTEGRACIÓN AL DEPORTE
}

\section{ADAPTED PADEL PRACTICE, A MODEL OF INTEGRATION TO THE SPORT}

\author{
Gabriel Barbero Montesinos ${ }^{(1)}$ y Gabriel Barbero Reyes ${ }^{(2)}$ \\ ${ }^{(1)}$ Director Deportivo Club Deportivo Campolivo de Úbeda \\ ${ }^{(2)}$ Maestro Especialista en Educación Física. CEIP Sebastián de Córdoba (Úbeda)
}

\begin{abstract}
RESUMEN: Se encuentra mayoritariamente asumido que el deporte puede contribuir a desarrollar valores, además de generar beneficios físicos, psicológicos y de carácter educativo. No obstante, no toda actividad deportiva desarrollada de cualquier modo, los origina siempre. Es necesario que se den una serie de condicionantes. De hecho, la práctica tan extendida hoy en día como es el pádel, el deporte amateur mas practicado justo por detrás del deporte rey como es el fútbol, fomentan valores no desarrollados hasta ahora para la integración de un discapacitado. En la presente comunicación, trataré de orientar mediante los recursos necesarios la práctica del pádel como elemento integrador para los discapacitados.
\end{abstract}

Palabras clave: Pádel, deporte adaptado, valores sociales, estrategias en la práctica

\begin{abstract}
Is mostly assumed that sport can contribute to develop values, as well as physical, psychological and educational benefits. However, not all sports activity in any case, originates them always. We need to give a series of conditions. In fact, the very widespread practice today as we paddle, amateur more practiced just behind Sport Sport King such as football, promoted values not developed so far for the integration of a disability. In this communication, I will try to guide through the resources the practice of the paddle as integrative element for the disabled.
\end{abstract}

Key words: Paddle, sport adapted, social values, strategies in practice.

Barbero Montesinos, G. y Barbero Reyes, G. (2011). Práctica de pádel adaptado, un modelo de intergración en el deporte. Espiral. Cuadernos del Profesorado, 4(8), 69-76. Disponible en: http://www.cepcuevasolula.es/espiral. 


\section{1.- INTRODUCCIÓN}

En los últimos años el pádel ha experimentado un incremento más que notable. Hoy día ya es una realidad extendida en sus diferentes áreas de influencia, antes solo era exclusividad de los clubes más elitistas, en determinados entornos y con la exclusividad de una clase social alta. Por suerte, se ha expandido y ha llegado a todas las familias siendo un deporte divertido, fácil de practicar y abierto a cualquier edad o sexo.

Cada vez es más notable la construcción de pistas de pádel en las infraestructuras deportivas de todas las ciudades, residenciales y apartamentos, por lo que conlleva la creación de escuelas, demanda de clases y monitores, incluso las familias lo incluyen entre sus alternativas de ocio y recreación.

Además los niños lo eligen como deporte principal dentro de sus actividades extraescolares, apuntándose a escuelas municipales o escuela de club, lo que formará parte de su formación personal. Por todo ello, y el continuo aumento en el número de participantes, parece indudable que el pádel amateur tiene el futuro más que garantizado

Por otro lado, que duda cabe que si como concepto de deporte nos limitásemos a la imagen y enfoque que muestran los medios de comunicación, relativos al deporte espectáculo, profesional de alto rendimiento, que acapara casi íntegramente, evidentemente atribuirle ciertas bondades pedagógicas y formativas de cara al desarrollo y maduración de todos sus participantes, quedaría fuera de lugar. Sin embargo, debemos de ser conscientes de que ese modelo de deporte supone una dimensión, un subconjunto o enfoque concreto dentro de la diversidad que engloba la concepción del deporte, ya que ahí no acaba todo, debemos de concienciarnos de que un sector de la población es discapacitada y necesita unas necesidades especiales para la práctica deportiva.

\section{2.- CLASIFICACIÓN Y NIVELES PARA DISCAPACITADOS}

Son varias las formas en que se pueden agrupar las distintas discapacidades. Hay autores que las dividen según sus incidencias en el aprendizaje, otros los clasifican por la edad de aparición y otros por sus trastornos. En síntesis, según las características que toma como predominante cada autor podemos encontrar tantas clasificaciones como autores se han dedicada a esta tarea.

Las más descriptivas y clara en su agrupación es la que propone el grupo de trabajo de discapacitados según la Sociedad Argentina de Pediatría (2003), que las clasifica en relación a su perdida funcional frente a su entorno.

1) Discapacidades motoras:

Es la persona que tiene una disminución motriz que le impide, por distintos factores, el manejar su cuerpo con total autonomía.

Dentro de las discapacidades motoras podemos clasificarlas en:

- No ambulatorias

- Semi-ambulatorias

- Ambulatorias (trastorno motor, sin afectar el tren inferior)

O también conforme a cuáles o cuántos miembros estén afectados (cuadripléjico, parapléjico, hemipléjico, etc.)

2) Discapacidades sensoriales:

Como su nombre lo explica, el discapacitado sensorial es la persona que se encuentra privada o con una disminución de alguno de sus sentidos (vista, habla, oído, etc.) haciendo que, a pesar de tener una total autonomía de su cuerpo, se siente dificultoso su relación con el exterior por la dependencia que en algunos casos puede creerse.

3) Discapacidades intelectuales:

La discapacidad mental es sumamente amplia y sin entrar en un análisis medico al respecto, podemos decir que es la que sufre toda persona que tiene una disminución de sus facultades mentales o intelectuales. 
4) Discapacidades viscerales:

Dentro de la clasificación efectuada tenemos como última la discapacidad visceral, tal vez la menos conocida en su acepción y que es la de aquellas personas que debido a alguna deficiencia en su aparato físico, le ocasiona la imposibilidad de desarrollar sus capacidades con total normalidad (por ejemplo el cardiaco, diabetes, asmático). Es decir personas que sin tener ninguna discapacidad intelectual, sensorial o motora, no pueden desarrollar su potencial con total plenitud.

Todas las discapacidades se dividen en función de su grado:

- Leves

- Moderadas

- Severas

- Profundas

Dicha categorías están basadas en la habilidad del alumno y no en su discapacidad física funcional según Baccigalupo (2008).

Principiantes: Niveles 1 y 2

$>$ Aquel jugador que no ha tenido experiencia en deportes de raqueta o su relación con los deportes en general sea muy elementales.

$>$ Dificultad en las habilidades básicas de coordinación general.

$>$ Se utilizará material adaptado (palas de lycra, de plástico, pelotas de foam, indiacas, pompones, etc.)

Enseñanza: Se persigue con ejercicios y de forma progresiva, con trabajos sobre la coordinación, desplazamientos, golpeos, precisión, etc., no se persiguen fines terapéuticos, sino también recreativos.

Competición: Los partidos se pueden jugar con reglas totalmente adaptadas y con un acompañamiento de pie.

\section{Intermedio: Nivel 3}

$>$ Aquel jugador que cuenta con alguna experiencia en deportes de raqueta y/o en pádel.

$>$ Sus habilidades básicas y coordinación general algo desarrolladas.

$>$ Juzga la distancia de la bola, aprende a moverse hacia ella.

Enseñanza: Basado en el desarrollo mediante juegos recreativos de ocio y disfrute.

Competición: Los partido se juega adaptando algunas reglas. Ej.: El saque no necesariamente a de entrar en el cuadrado correspondiente o agregar mas más botes.

Avanzado: Nivel 4.

$>$ Aquel jugador con experiencias en deportes y en pádel.

$>$ Su coordinación y manejo del espacio es óptimo.

Enseñanza: Aprendizaje de los golpes básicos del pádel, mediante repetición.

Competición: Los partido se juega con el reglamento oficial de la FIP y pueden jugar en equipo armando previamente táctica de juego. Adicionalmente a la enseñanza se le puede incorporar algún tipo de entrenamiento.

Juego integrado: Se pueden jugar uno en silla de ruedas y el otro no.

\section{3.- REGLAMENTO DE PADEL ADAPTADO}

Basado en el Reglamente de la Asociación Pádel Para Todos, y utilizando el Reglamento Oficial de la FIP (Federación Internacional de Pádel), con algunas adaptaciones, como son:

\section{- Regla 1. Posición de los jugadores}


En los niveles 2 y 3 y silla de ruedas no se efectuarán cambios de lado, tal y como dicta el reglamento, ya que la duración del partido no lo justifica, solo se efectuará cambio de lado, si la posición del sol u otra causa lo justificara.

En pádel en silla de ruedas, si la pista planteara dificultades importantes para efectuar el cambio de lado de los jugadores, será potestad del juez árbitro establecer la frecuencia de los cambios.

FIP: El tiempo máximo de descanso en los cambios de lado será de 90 segundos.

En pádel adaptado, no hay tiempo de descanso en niveles 2 y 3.

- Regla 2. Elección de lado y del servicio. Sorteo inicial

Regla anulada en niveles 2 y 3

\section{- Regla 3. El saque o servicio}

FIP: El que lo ejecute (el servidor) deberá estar en el momento de inicio del servicio con ambos pies detrás de la línea de saque.

Nivel 2: El jugador que efectúa el saque no tendrá una posición determinada ni obligatoria, bastara con que, desde cualquier situación en la que se encuentre, consiga, con o sin ayuda, pasar la pelota a campo contrario. Sin que en este haya una zona delimitada de saque, por tanto el saque se considera efectuado con el pase de la pelota a cualquier zona del campo.

Nivel 3: El servidor deberá estar en el momento del inicio del servicio entre la línea central y la pared lateral, sin que se considere falta de saque el hecho de pisar la línea de fondo. El saque se considera efectuado cuando la pelota se lanza en diagonal hacia el recuadro de saque contrario.

Silla de ruedas: El jugador que efectúa el saque deberá tener en el inicio del servicio la rueda o ruedas delanteras de la silla por detrás de la línea de saque, y se colocará entre la línea central y la pared lateral.

Sillas de ruedas: en el saque el jugador deberá golpear la pelota por debajo de sus hombros.

\section{- Regla 4. Falta de servicio}

FIP: Luego de servida, la pelota bota fuera del área de servicio del restador, la que incluye las líneas que la delimitan (las líneas son buenas).

Regla no valida para el nivel 2 que no delimita zona de resto.

En nivel 3 y silla de ruedas, como mínimo el primero de los botes permitidos debe producirse en el área de servicio

\section{- Regla 5. Orden de saque}

En niveles 2 y 3 se establece modificación en la puntuación, esta se realiza por tantos (como en el tie break) por tanto las ordenes de saque las establecerá el monitor, tendiendo a que sean 2 saques por jugador.

\section{- Regla 6. El resto o devolución} para hacerlo.

Nivel 2: No se establece restador, la pelota la restara aquel jugador que tenga más facilidad

Nivel 3.- no se establece reglamento sobre la limitación del número de botes

Sillas de ruedas: el jugador que resta deberá esperar a que la pelota bote dentro de su área de servicio y deberá golpearla antes del tercer bote.

\section{- Regla 7. Saque que toca a un jugador}

Nivel 3 y sillas de ruedas: si al jugador que resta o a su compañero les golpea la pelota en el cuerpo o en la silla, o la tocaran con la pala, antes de que hubiera botado, estando dentro de la zona de saque, se considerará tanto del jugador al servicio.

\section{- Regla 8. Puntuación}

La puntuación oficial de la FIP, solo valida para nivel 4, en el resto de los niveles la puntuación será como la de un tie break, sin que sea necesaria la diferencia de 2 tantos, el limite de puntuación se marca de antemano. 


\section{- Regla 9. Peloteo en juego}

Regla valida para todos los niveles, exceptuando la limitación de números de botes en el suelo, no valido en los niveles 2, 3 y sillas de ruedas donde el número de botes es mayor, dos en el caso de sillas de ruedas.

Sillas de ruedas: Si las nalgas del jugador que golpea la pelota no están en contacto con el asiento de la silla de ruedas. Si el jugador cae de la silla de ruedas, después de dar a la pelota, debe volver a la silla antes de efectuar el siguiente golpe. El compañero del que se cayó puede seguir disputando los puntos, mientras éste se vuelve a sentar.

\section{- Regla 10. Sustitución de pelotas}

Sillas de ruedas: Se podrá jugar con 6 pelotas si las condiciones así lo aconsejan.

\section{4.- COMPARACIÓN ENTRE TENIS ADAPTADO Y PADEL ADAPTADO}

- La pista de pádel contiene paredes, las paredes son un aliado.

$>$ El desarrollo del punto en pádel, dura más que en el tenis.

$>$ Con los revotes de pared, en la practica del pádel, se alcanzan bolas mas difíciles.

- En pádel, siempre se juega por parejas.

$>$ En la practica de deportes de raqueta por parejas, siempre es mas fácil jugar, se apoya un compañero a otro ("acércate" - "aléjate") y puede hace jugadas.

$>$ En parejas, pueden anticiparse a la jugada uno de los dos compañeros para ganar el punto.

- El pádel es un deporte agradecido.

$>$ No se tiene temor al ridículo, ya que más o menos es fácil de pasar la pelota de un campo a otro.

$>$ Los espacios del la pista de juego, en pádel (20x10m) son mucho mas pequeños que en tenis $(24 \times 11 \mathrm{~m})$

$>$ Se equiparan los niveles para jugar.

$>$ El principiante lo disfruta desde los inicios.

$>$ El saque en pádel, es mucho mas fácil y asequible que en tenis.

\section{5.- DIAGNÓSTICO Y EVALUACIÓN}

Es necesario para este tipo de práctica deportiva para discapacitados, sobre todo para discapacitados intelectuales, se realice un diagnostico inicial y una evaluación semestral integral a cada sujeto que realice la practica, para ello es necesario un equipo interdisciplinario con psicólogos y personal cualificado.

En ese diagnostico tendremos que valorar:

- Técnica deportiva:

$>$ Coordinación fina y gruesa.

$>$ Desplazamiento.

$>$ Aprender a dirigir la pelota tras el impacto aun área determinada y pueda variar la dirección.

$>$ Juzgar la distancia de la pelota y aprender a moverse hacia ella.

- Comportamiento motriz:

$>$ Desarrollo de sus habilidades motoras básicas (correr, lanzar, saltar, recibir, caminar, manejo de objetos, saltar con una pierna, saltar con la otra).

$>$ Desarrollo de la colocación del cuerpo.

$>$ Adquisición de una conciencia interna del cuerpo y de las partes corporales entre sí.

- Aspectos sociales: 
$>$ Integración con el grupo.

$>$ Relación con los profesores.

$>$ Trabajo en equipo y colaboración.

$>$ Comunicación.

- Aspectos psicológicos:

$>$ Concentración.

$>$ Motivación.

$>$ Autoconfianza.

$>$ Comprensión.

\section{6.- CÓMO DESARROLLAR UNA PRÁCTICA DE PÁDEL ADAPTADO}

Existen dos modalidades de pádel adaptado, como son: en silla de ruedas y discapacidad intelectual.

\section{Pádel en silla de ruedas}

La silla ha evolucionado a lo largo del tiempo, en cuanto a materiales y elementos.

- Características de una silla de ruedas:

> Para el pádel se recomienda las dos ruedas delanteras y un "anti tip" corto para que en el momento de estar en el fondo, no choquemos con la pared.

$>$ Las sillas son de tamaño y altura al jugador.

$>$ Se han ido incorporando ángulos a las ruedas para mayores giros y movilidad en la pista.

$>$ Es imprescindible unos cinturones atados a las rodillas, para que la la silla y la persona sea "uno".

$>$ El ideal son sillas de carbono, que sean muy ligeras. Ej. 6 kg.

- Características de juego en la pista:

$>$ La silla nunca se mueve para atrás, siempre hay que estar de cara a la pelota y en caso de ser necesario se usan giros.

$>$ Es mucho más dinámico estar de frente y utilizar giros.

$>$ Estratégicamente, los jugadores no deben de ubicarse ni muy adelante ni muy atrás, sino que en $3 / 4$ de su lado de pista.

$>$ Se utiliza mucho el bote pronto.

\section{Pádel en discapacidad intelectual}

En esta otra modalidad de pádel adaptado, lo que se debe de llegar a genera, sería:

- Aprendizaje por medio del deporte y que puedan ser trasladados a la vida cotidiana del individuo.

- Además hay que tratar de estimular el proceso de crecimiento y desarrollo.

- Estimular los elementos que intervienen en el desarrollo psicomotor como esquema corporal, lateralidad, equilibrio, nociones espacio temporales, todo ello conseguido a través del deporte.

\section{Técnica de los golpes}

La técnica de los golpes en estas dos modalidades son similares, teniendo en cuanta las posibilidades de cada jugador, y los elementos a tener en cuenta como son la silla por un lado y el agarre de la pala por otro.

- Se empuñara la pala con mango corto

- Se iniciaran todos los golpes sin pelota 
- Se incorporara la pelota pero con recorrido corto de movimiento en todos los golpes básicos de pádel.

- Si el alumno no tiene la suficiente fuerza, puede ayudarse con las dos manos.

- Se realizarán pocas repeticiones, incorporando juegos divertidos a la práctica.

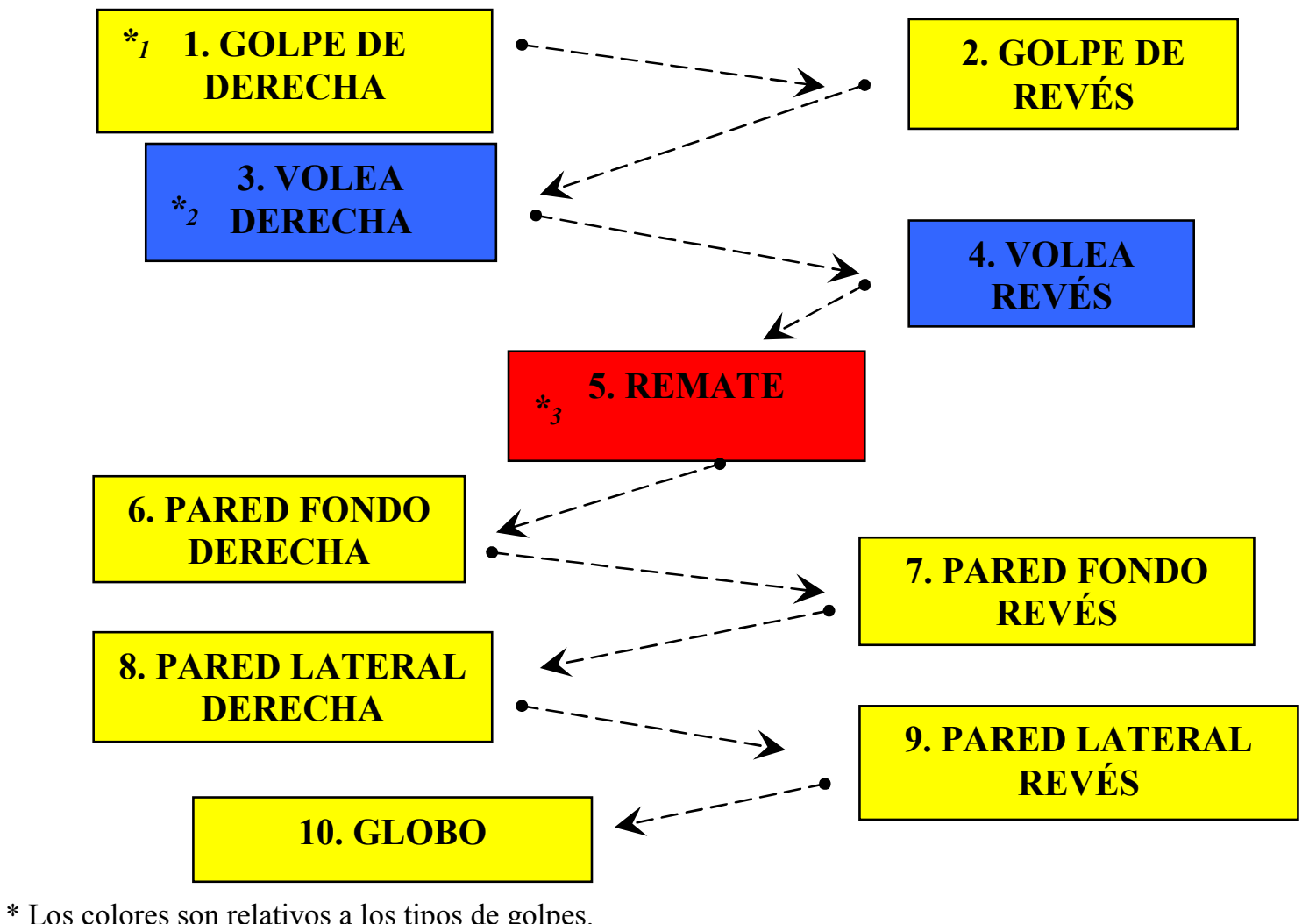

* Los colores son relativos a los tipos de golpes.

Figura 2.- Esquema de progresión de enseñanza de los golpes

En el esquema anterior (figura 2) se ha reseñado de 1 a 11 el orden en el que se deben ver los golpes, siempre y cuando empecemos de cero o en una clase de iniciación a este deporte. Este orden no se realiza así por azar, sino que algunos autores proponen esta secuenciación metodológica en la cual se han llevado a cabo estudios sobre el tema según González-Carvajal (2006).

En los mismos se han llegado a las siguientes conclusiones:

- Realizar aprendizaje progresivo en sencillez- dificultad:

$1^{\circ}$ Golpes "básicos" (derecha y revés): Son golpes naturales, son uno de los golpes más frecuentemente utilizados, el bote es uniforme y resta velocidad y fuerza a la bola.

$2^{\circ}$ Golpes sin bote previo (voleas y remate): Golpeamos la bola directamente, lo cual, deducimos que viene con más velocidad y fuerza causando menos control sobre la bola.

$3^{\circ}$ Golpes de paredes de fondo: En este caso metemos la dificultad de un rebote. Éste es uniforme, ya que rebota siempre hacia delante.

$4^{\circ}$ Golpes de pared lateral: Estamos ante un caso parecido al anterior, aunque existen diferencias en cuanto al rebote que no es uniforme y despista más.

$5^{\circ}$ Golpe táctico: En este caso nos referimos al globo, es un golpe de recurso, aunque esencial en la práctica del pádel.

- Siempre se enseñan los golpes de derecha antes que los de revés, ya que es más "natural".

- El saque se realiza junto a la derecha o el revés. 


\section{7.- DIFERENCIAS EN LOS TIPOS DE GOLPES ADAPTADOS}

$*_{1}$ Fondo: Se realizan en la zona 1 . El golpe va de atrás adelante para que la bola suba y supere la red. Golpes defensivos: Golpe de derecha y de revés, pared de fondo y paredes laterales y globo.

${ }_{2}$ Intermedio: Se realizan en la zona 2 . Son golpes de transición y su característica principal es la seguridad, es decir, no se pueden fallar: Volea de derecha y de revés.

${ }_{3}$ Red: Se realizan en zona 3. Golpe de arriba abajo, ya que estamos cerca de la red lo que necesitamos es que la bola baje lo antes posible al suelo. Golpes ofensivos: Remate (figura 3)

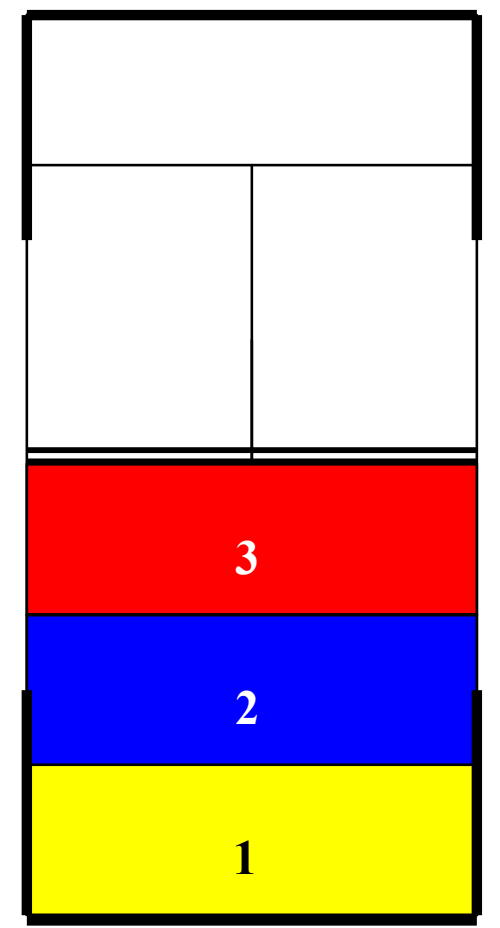

Figura 3.- Diferencias en los tipos de golpes adaptados.

\section{8.- REFERENCIAS BIBLIOGRÁFICAS}

Baccigalupo, C. (2008). Pádel Adaptado. Buenos Aires: Fundación Deporte con Discapacidad

González-Carvajal, C. (2006). Escuela de Pádel: Del Aprendizaje a la Competición Amateur. Madrid: Ediciones Tutor.

VV.AA. (2005). Pádel para todos. Madrid: Asociación de Pádel para todos.

VV.AA. (2011). Reglamento Oficial FIP. Madrid: Federación Internacional de Pádel.

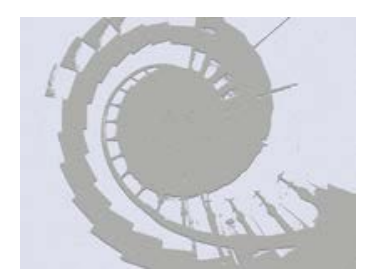

